\title{
Evidence for obtaining a second successive semen sample for intrauterine insemination in selected patients: results from 32 consecutive cases
}

\author{
Alejandra Ortiz ${ }^{1}$, Rita Ortiz ${ }^{1}$, Evelyn Soto ${ }^{1}$, Jonathan Hartmann ${ }^{2}$, Alejandro Manzur ${ }^{1}$, Marcelo Marconi ${ }^{1,2}$ \\ 'Human Reproduction Unit, ${ }^{2}$ Urology Department, Pontificia Universidad Católica de Chile, Santiago, Chile
}

Objective: The goal of this study was to compare the semen parameters of two successive samples obtained within an interval of less than 60 minutes from patients planning to undergo intrauterine insemination (IUI) whose first samples exhibited low semen quality.

Methods: Thirty-two consecutive patients were enrolled in the study. On the day of IUI, the semen analysis of the samples initially presented by all patients met at least two of the following criteria: sperm concentration $<5 \times 10^{6} / \mathrm{mL}$, total sperm count $<10 \times 10^{6}$, progressive sperm motility $(a+b)$ in the native sample $<30 \%$, and total motile sperm count (TMSC) $<4 \times 10^{6}$. A successive semen sample was obtained no more than 60 minutes after the first sample.

Resultts: Compared to the first sample, the second exhibited significantly $(p<0.05)$ improved sperm concentration, TMSC, progressive motility, and vitality. Regarding TMSC, the most critical parameter on the day of IUI, 23 patients (71.8\%) improved it, while nine (28.2\%) displayed poorer outcomes.

Conclusion: In defined cases, requesting a second successive ejaculate on the day of insemination may result in a high percentage of cases in an improvement of the quality of the sample.

Keywords: Intrauterine insemination; Semen; Sperm count

\section{Introduction}

It is generally presumed that sperm concentration significantly decreases when the abstinence period is reduced, especially if the samples are collected at an interval of 24 hours or less [1,2]. Operating within this paradigm, the World Health Organization (WHO) recommends an abstinence period of 2 to 7 days [3]. The most recent edition of the WHO manual states that if a new sample is needed, the same abstinence period ( 2 to 7 days) is recommended, with no mention of the possibility of obtaining a second sample immediately af-

Received: Feb 18, 2016 · Revised: Apr 10, 2016 · Accepted: Apr 27, 2016

Corresponding author: Marcelo Marconi

Urology Department, Pontificia Universidad Católica de Chile, Marcoleta 350,

Santiago, Chile

Tel: +56-9-79685115 Fax: +56-2-23543468 E-mail: mmarconi@andro.cl

This is an Open Access article distributed under the terms of the Creative Commons Attribution Non-Commercial License (http://creativecommons.org/licenses/by-nc/4.0/) which permits unrestricted non-commercial use, distribution, and reproduction in any medium, provided the original work is properly cited. ter the first [3]. It has been established that sperm concentration and motility are highly variable, which explains the fact that patients with previous acceptable ejaculate analyses may present on the day of intrauterine insemination (IUI) with a poor-quality or extremely poorquality sample. No clear guidelines exist regarding the preferable course of action in such scenarios, with possible plans including cancellation of the procedure, performing the insemination with any samples of motile sperm that are obtained from the sample, or asking for a second sample after no more than 60 minutes of abstinence.

Interestingly, some studies have reported improved semen parameters in second successive ejaculate samples in infertile patients. Juarez-Bengoa et al. [4] and Bar-Hava et al. [5] observed increased sperm concentrations in successive ejaculation samples from oligozoospermic patients. However, other researchers have not observed statistically significant differences in sperm concentrations in the same settings [6,7]. Regarding other parameters, significant improve- 
ments in progressive sperm motility $(A+B)$ and total motile sperm count (TMSC) have been reported in second successive semen samples $[4,8,9]$. The totality of this evidence may be highly relevant in clinical settings, since, in many cases, patients with a low sperm concentration and/or low motility may be excluded from basic reproductive medicine treatments, such as IUI, due to a low TMSC. No consensus exists regarding whether to request a second successive sample in cases of low semen quality. The objective of this study was there-

Table 1. Clinical characteristics and previous semen analyses of the 32 patients enrolled in the study

\begin{tabular}{lc}
\hline Parameter & Value \\
\hline Age (yr) & $38(27-47)$ \\
Andrological features & \\
No significant andrological history & 19 \\
Idiopathic oligo-astheno-teratozoospermia & 6 \\
Previously operated palpable varicocele & 2 \\
History of undescended testis & 2 \\
Male accessory gland infection (treated) & 2 \\
History of testicular tumor & 1 \\
Semen analysis & \\
Volume (mL) & $2.6 \pm 1.6$ \\
pH & $7.7 \pm 0.3$ \\
Sperm concentration (million/mL) & $36.2 \pm 50.5$ \\
Total sperm count (million) & $69.5 \pm 79.5$ \\
Progressive motility (\%) & $44.7 \pm 18.3$ \\
Normal morphology (\%) & $5.1 \pm 3.8$ \\
\hline
\end{tabular}

Values are presented as mean (range), number, or mean \pm standard deviation.

A

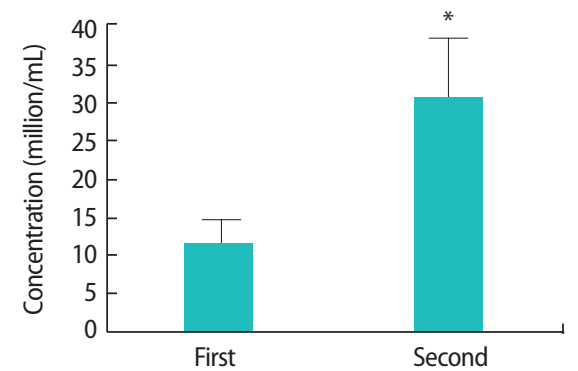

C

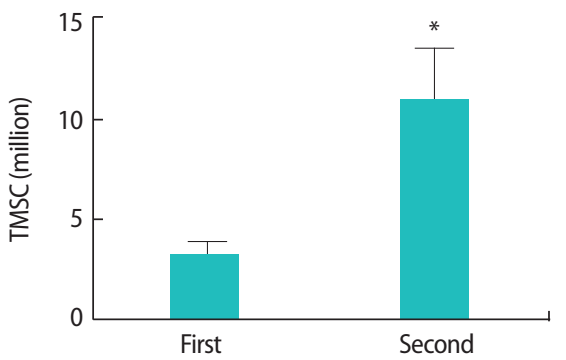

fore to compare the semen parameters of two successive ejaculations with an interval of less than 60 minutes, in patients who attended our reproductive unit for an IUI procedure and exhibited low semen quality in their first sample.

\section{Methods}

Thirty-two consecutive patients (mean age, 38 years; range, 27-47 years) who were treated in our facility from June 2012 to May 2015 were enrolled in the study. All patients underwent a basic andrological evaluation, which included two semen analyses according to the WHO manual (Table 1) [3]. On the day of the IUI, the median abstinence time was 3 days (range, 1-5 days). At least two of the following criteria were observed in all patients included in this study in the analysis of their first semen sample: (1) sperm concentration $<5 \times 10^{6} / \mathrm{mL}$, (2) total sperm count $<10 \times 10^{6}$, (3) progressive sperm motility $(a+b)$ in native sample $<30 \%$, and (4) TMSC $<4 \times 10^{6}$.

These inclusion criteria were based on the data published by Dickey et al. [10], who concluded that these values represent the threshold fertility levels for initial sperm specimens intended for use in IUI, because samples under these levels exhibited significantly lower pregnancy rates [10].

We defined successive semen samples as those obtained no more than 60 minutes after the first sample. All samples were analyzed according to the WHO standards [3]. In order to obtain the TMSC, a concentration gradient was performed. Strict morphology was not evaluated on the day of insemination in either of the two samples, as it was

B

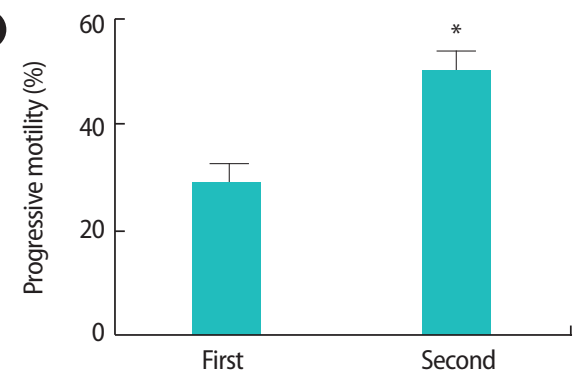

D

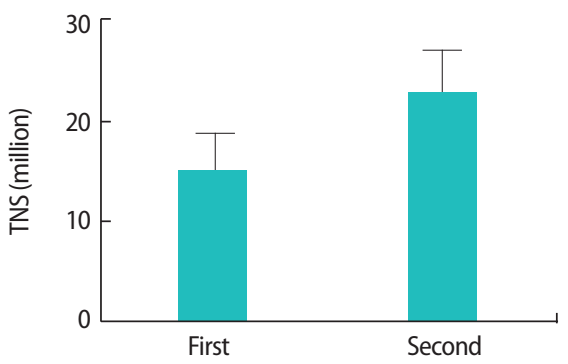

Figure 1. The second successive sample exhibited significantly improved ( $p<0.05$; paired samples $t$-test) values for sperm concentration $(11 \pm 17.8$ vs. $30 \pm 46.5$ million/mL) (A), progressive motility ( $29 \% \pm 17.7 \%$ vs. $50 \% \pm 21.6 \%)(B)$, and total motile sperm count (TMSC, $3.38 \pm 3.8$ vs. $11.5 \pm 15.4$ million) (C). A trend towards improvement was observed in the total number of sperm (TNS) (14.8 \pm 21.4 vs. $22.5 \pm 25.9$ million) (D), although this trend did not reach statistical significance. ${ }^{*} p<0.05$. 
evaluated in the previous semen analysis of each patient (Table 1).

This study was approved by the Institutional Review Board of the faculty of medicine, Pontiificia Universidad Catolica de Chile.

Statistical analysis was performed using the GraphPad Prism statistical software (San Diego, CA, USA), and $p$-values $<0.05$ were considered to indicate statistical significance.

\section{Results}

Clinical features and semen analyses of the 32 patients enrolled in the study are in Table 1.

The second semen samples exhibited significant improvements in comparison to the first samples ( $p<0.05$, paired samples $t$-test) in terms of sperm concentration, TMSC and progressive motility (Figure 1). More specifically, 24 patients (75\%) exhibited an improved sperm concentration, while eight patients (25\%) showed a diminished sperm concentration; 23 patients (71.8\%) exhibited an improved TMSC, while nine (28.2\%) showed a poorer TMSC; and 28 patients $(87.5 \%)$ showed improved progressive motility while four patients (12.5\%) had poorer values for this parameter. The semen volume was significantly lower $(p=0.001)$ in the second sample $(1.8 \pm 0.9 \mathrm{~mL}$ vs. $1.2 \pm 0.8 \mathrm{~mL}$ ); this factor may explain the observation that although the total number of sperm (TNS) tended to increase in the successive ejaculate sample, this trend did not reach statistical significance ( $p>0.05$, paired samples $t$-test) (Figure 1). Three pregnancies (pregnancy rate, $9.37 \%$ ) were achieved after the $32 \mathrm{IUI}$ procedures.

\section{Discussion}

Historically, an abstinence period has been suggested both for semen analysis and for collecting samples for reproductive medicine procedures. The most recent version of the WHO manual recommends 2 to 7 days [3]. It has been suggested that a shorter abstinence period would negatively impact the sperm concentration and/or total sperm count [1,2]; however, the evidence supporting this assumption is not completely clear [11], especially regarding successive samples obtained no more than minutes or hours after the first sample. Previous reports have suggested that the sperm concentration in a second successive sample, obtained between 2 to 4 hours after the first sample, is at least as high as the initial concentration $[5,7]$. Other studies have demonstrated that second successive samples exhibited significantly improved progressive motility and reduced sperm DNA fragmentation $[4,6,9]$. The totality of this evidence becomes extremely relevant when treating a couple with a male partner who has a low sperm count and/or decreased progressive motility, since these facts affect the type of treatment they are counseled to undergo $[12,13]$. Our results demonstrated that in pa- tients who presented with a sperm concentration $<5$ million $/ \mathrm{mL}$ in the native sample, a total sperm count $<10$ million, progressive motility $<30 \%$, and/or a TMSC $<4$ million, a second successive sample has a $71.8 \%$ chance of improving the above factors. Regarding the diagnosis and prognosis, this study did not evaluate whether the improvement observed in the second successive sample was repeatable over time, and therefore we cannot recommend this intervention as a standard option for patients with poor ejaculate quality who undergo IUI. Also, as pointed out by other researchers, it is not clear whether such results occur only in patients with low-quality semen samples or reflect a more general phenomenon $[4,8]$. However, we believe that our study demonstrates that, in specific cases, a second successive sample may help, especially considering that it is a noninvasive procedure and that the extra cost is low.

During the emission phase of ejaculation, the different portions of the epididymis, especially the tail, contract in order to expel mature sperm to the deferent duct [14]. Traditionally, it is thought that after an ejaculation, the epididymal tail is depleted of motile sperm, and that a certain time ( 2 days) must pass for this portion of the epididymis to be refilled with motile sperm [15]. Clinically, this agrees with evidence showing that patients who ejaculate on a daily basis exhibit decreased sperm concentration [1,2]. However, our study and others suggest that a successive semen sample obtained 1 to 4 hours after the first does not necessarily exhibit reduced sperm concentration or TMSC, and may even have better parameters [4,7,9].

Regarding sperm concentration, we think that at least two possible explanations may exist for our results. First, a mean of 200 million sperm are stored in the human epididymis, half of them in the head and body of the gland, and the rest in the tail [15]. In some cases, the first ejaculation may not completely empty the more proximal part of the epididymis (the tail). This has been observed in other species, such as rabbits, in which more than one ejaculation may be necessary to clear out the tail of the epididymis and the vas deferens $[16,17]$. The second possible explanation is that, as in other species, when a demand emerges for more frequent ejaculations, the transit speed through the epididymis is accelerated, resulting in increasing sperm concentrations in successive samples [18].

As in previous reports [6,8], the patients in our case series had significantly better progressive sperm motility in the second successive sample. This phenomenon may be secondary to the fact that the first set of sperm had been present for a longer period of time in the epididymal tail, affecting its overall motility. The second sperm set (in the successive ejaculate) passed through the epididymal tail more quickly, giving it an increased motility potential. This may be explained by the fact that the maximum sperm motility is acquired in the more proximal part (near the tail) of the epididymal body; in fact, in humans, sperm motility is $27 \%$ lower in the tail than in the more 
proximal part of the epididymal body [19]. After emptying the tail in the first ejaculation, sperm cells coming from the body of the epididymis are ejaculated in successive samples, which explains their improved motility [20].

The main limitations of our study are the small number of patients, the lack of information regarding variation in the strict morphology of the two successive samples, and the absence of a control group to compare pregnancy rates.

In conclusion, obtaining a second successive semen sample in certain cases is a rational course of action that may lead to improvements in the quality of the sample in the majority of patients. It is especially useful in men who present on the day of IUI with a low-quality sample, allowing the procedure to be performed with a higher number of motile sperm. More studies are needed to confirm these results.

\section{Conflict of interest}

No potential conflict of interest relevant to this article was reported.

\section{References}

1. van Duijn C Jr, Freund M. The relationship between some seminal characteristics and ejaculation frequency in the human male. Eur J Obstet Gynecol 1971;1:167-74.

2. Carlsen E, Petersen JH, Andersson AM, Skakkebaek NE. Effects of ejaculatory frequency and season on variations in semen quality. Fertil Steril 2004;82:358-66.

3. World Health Organization. WHO laboratory manual for the examination and processing of human semen. Geneva: World Health Organization; 2010.

4. Juarez-Bengoa A, Guadarrama-Garcia LF, Diaz-Perez Mde L. Potential treatment of infertility with second ejaculate. Ginecol Obstet Mex 2010;78:29-36.

5. Bar-Hava I, Perri T, Ashkenazi J, Shelef M, Ben-Rafael Z, Orvieto R. The rationale for requesting a second consecutive sperm ejaculate for assisted reproductive technology. Gynecol Endocrinol 2000;14:433-6.

6. Hussein TM, Elariny AF, Elabd MM, Elgarem YF, Elsawy MM. Effect of repeated sequential ejaculation on sperm DNA integrity in subfertile males with asthenozoospermia. Andrologia 2008; 40:312-7.

7. Zhai DM, Li MJ, Jiang L, Qin AP, Li LM, Hang F. Results of request- ing a second consecutive sperm ejaculate on the day of oocyte pick-up in assisted reproductive technology. Zhonghua Nan Ke Xue 2011;17:418-21.

8. Tur-Kaspa I, Maor Y, Levran D, Yonish M, Mashiach S, Dor J. How often should infertile men have intercourse to achieve conception? Fertil Steril 1994;62:370-5.

9. Tur-Kaspa I, Dudkiewicz A, Confino E, Gleicher N. Pooled sequential ejaculates: a way to increase the total number of motile sperm from oligozoospermic men. Fertil Steril 1990;54:906-9.

10. Dickey RP, Pyrzak R, Lu PY, Taylor SN, Rye PH. Comparison of the sperm quality necessary for successful intrauterine insemination with World Health Organization threshold values for normal sperm. Fertil Steril 1999;71:684-9.

11. Mayorga-Torres BJ, Camargo M, Agarwal A, du Plessis SS, Cadavid AP, Cardona Maya WD. Influence of ejaculation frequency on seminal parameters. Reprod Biol Endocrinol 2015;13:47.

12. Strandell A, Bergh C, Soderlund B, Lundin K, Nilsson L. Fallopian tube sperm perfusion: the impact of sperm count and morphology on pregnancy rates. Acta Obstet Gynecol Scand 2003; 82:1023-9.

13. ESHRE Capri Workshop Group. Intrauterine insemination. Hum Reprod Update 2009;15:265-77.

14. Vignozzi L, Filippi S, Morelli A, Luconi M, Jannini E, Forti G, et al. Regulation of epididymal contractility during semen emission, the first part of the ejaculatory process: a role for estrogen. J Sex Med 2008;5:2010-6.

15. Amann RP, Howards SS. Daily spermatozoal production and epididymal spermatozoal reserves of the human male. J Urol 1980;124:211-5.

16. Prins GS, Zaneveld LJ. Radiographic study of fluid transport in the rabbit vas deferens during sexual rest and after sexual activity. J Reprod Fertil 1980;58:311-9.

17. Kihara K, Sato K, Ando M, Azuma H, Oshima H. Antegrade and retrograde fluid transport through the vas deferens. Am J Physiol 1995;269:R1197-203.

18. Ambriz D, Rosales AM, Sotelo R, Mora JA, Rosado A, Garcia AR. Changes in the quality of rabbit semen in 14 consecutive ejaculates obtained every 15 minutes. Arch Androl 2002;48:389-95.

19. Yeung $\mathrm{CH}$, Cooper TG, Oberpenning F, Schulze H, Nieschlag E. Changes in movement characteristics of human spermatozoa along the length of the epididymis. Biol Reprod 1993;49:274-80.

20. Cooper TG. The epididymis, sperm maturation and fertilisation. Berlin: Springer-Verlag; 1986. 